\title{
Review of: "Specific absorption rate reduction for sub-6 frequency range using polarization dependent metamaterial with high effective medium ratio"
}

Jianguo Zhang

Potential competing interests: The author(s) declared that no potential competing interests exist.

Concern about RF exposure to humans increases tremendously because of the fast growth in the usage of mobile phones. Hence, a reduction of radiation exposure from a mobile phone is highly recommended in the current research field. In this paper, the authors propose a multi-layered square-shaped metamaterial (MSM) structure, which can be employed in EM absorption reduction applications and meet the objectives of current research. Meanwhile, the work provides a comprehensive and technology-oriented perspective, which can help researchers better understanding the current state of the metamaterials, as well as the future efforts in this field. Furthermore, I believe this guidance will be timely for the development of metamaterials. 